\title{
8. Das Realismusproblem: Entitäten oder Strukturen ?
}

Wie im vorausgehenden Kapitel deutlich geworden sein dürfte, ist es nicht ohne weiteres möglich, die vom Stringansatz in seinen diversen Erscheinungsformen jeweils als basal angesetzten Entitäten in realistischer Weise als objekthafte Komponenten eines raumzeitlichen Geschehens zu deuten. Gibt es aber vielleicht andere Komponenten innerhalb der theoretischen Konstrukte, die sich im Gegensatz zu den jeweils postulierten basalen oder auch weniger basalen Entitäten in irgendeiner Weise realistisch deuten lassen? Gibt es irgendetwas im Stringansatz, dem realer ontischer Status zugesprochen werden kann? Allgemeiner gefragt: Wie steht der Stringansatz zum Realismus? Inwieweit leistet er ihm Vorschub? Und in welcher Weise setzt er ihn vielleicht sogar voraus? Was wäre, wenn er zwar den Realismus voraussetzt, letztlich aber nicht oder nur eingeschränkt mit ihm verträglich ist? - Für mögliche Antworten auf diese Fragen gelten gleichermassen die schon im vorausgehenden Kapitel vorgebrachten Vorbehalte hinsichtlich ihrer unabwendbaren Vorläufigkeit angesichts des aktuellen Standes der Theorieentwicklung im Stringansatz. Dennoch lassen sich vielleicht immerhin schon einige tentative Überlegungen hinsichtlich der Stellung des Stringansatzes zum Realismus anstellen:

Für eine grundsätzlich realistische Interpretation hinsichtlich der Aussagen empirisch bestätigter, erfolgreicher Theorien, den sogenannten "wissenschaftlichen Realismus", ${ }^{382}$ wird immer wieder ein zentrales Argument ins Feld geführt: das sogenannte "Erfolgsargument" bzw. "No-miraclesArgument". Dieses Argument besagt, dass der Erfolg einer Theorie, der vor allem in einer umfangreichen empirischen Bestätigung ihrer Vorhersagen zu sehen ist, aber längerfristig auch etwa technische Umsetzungsmöglichkeiten umfassen kann, nur dann nicht zum unerklärlichen Wunder wird, wenn die Aussagen dieser Theorie im wesentlichen wahr sind und wenn die von der Theorie beschriebene Dynamik und die von ihr postulierten Entitäten in irgendeiner Weise über ein Gegenstück in der Realität verfügen.

$382 \quad$ Siehe etwa Leplin (1984), Laudan (1981), Psillos (1999). 
"It would be a mircale, a coincidence on a near cosmic scale, if a theory made as many correct empirical predictions as, say, the general theory of relativity or the photon theory of light without what that theory says about the fundamental structure of the universe being correct or 'essentially' or 'basically' correct." (Worrall (1989) 101)

Ausser der Allgemeinen Relativitätstheorie, deren in mancher Hinsicht überraschende theoretische Vorhersagen zum Teil schon kurz nach ihrer Aufstellung empirisch bestätigt werden konnten, wird hier als Beispiel gerne die Quantenmechanik angeführt, die nicht nur über die vielfältigsten empirischen Bestätigungen verfügt und sich bisher als mit allen empirischen Daten in vollem Umfang verträglich erwiesen hat, sondern inzwischen sogar diverse technische Umsetzungen erfahren hat; und alles sieht danach aus, als ob diese Quantentechnologien in der Zukunft an Bedeutung gewinnen werden. Aus dem empirischen und zum Teil technologischen Erfolg solcher Theorien wird im Sinne des No-miracles-Arguments auf die Wahrheit (im korrespondenztheoretischen Sinne) oder zumindest die annähernde Wahrheit der von ihnen vorgebrachten, die Wirklichkeit betreffenden Aussagen geschlossen.

Für den Stringansatz könnte das No-miracles-Argument als Stützung seiner realistischen Interpretierbarkeit jedoch erst dann ins Feld geführt werden, wenn er sich insofern als erfolgreich erweisen sollte, dass er unabhängige empirische Bestätigungsinstanzen erhält: Bestätigungsinstanzen also, die unabhängig sind von dem empirischen Gehalt, der in den Stringansatz aus seinen Vorgängertheorien eingeflossen ist. Hier genügt die vermeintliche "Vorhersage" der Gravitation in gar keiner Weise. ${ }^{383}$

Aber auch eine empirisch und vielleicht sogar technologisch erfolgreiche Theorie ist hinsichtlich einer realistischen Interpretierbarkeit ihrer Aussagen immer noch erheblichen Unsicherheiten ausgesetzt: Dem No-miraclesArgument als Hauptstützpfeiler einer realistischen Interpretation unserer erfolgreichen Theorien stehen nämlich unsere Erfahrung mit der Wissenschaftsgeschichte entgegen. Immer wieder wurden recht erfolgreiche, $\mathrm{zu}$ ihrer Zeit in ausreichendem Masze empirisch bestätigte Theorien von Nachfolgern abgelöst, die zu überraschenden, mit den Implikationen ihrer Vorgänger unverträglichen, neuen Vorhersagen führten, welche in der

$383 \quad$ Vgl. Kap. 4.3. 
Folge schliesslich empirisch bestätigt werden konnten und somit die Vorgängertheorien als nicht empirisch adäquat herausstellten. Über diese Wissenschaftsumbrüche hinweg blieb zwar der empirische Gehalt der jeweiligen Vorgängertheorien weitgehend erhalten und wurde durch den mit den jeweils neuen Theorien hinzukommenden empirischen Gehalt erweitert. Was jedoch nicht erhalten blieb, waren oftmals die nomologischen und dynamischen Implikationen der jeweiligen Vorgängertheorien und insbesondere die von diesen Theorien postulierten Entitäten. Wissenschaftsumbrïche brachten also oft nicht zuletzt Ontologieumbrüche mit sich.

Angesichts dieser Erfahrungen mit der Wissenschaftsgeschichte ist es dann nicht fernliegend, auch für unsere aktuell als erfolgreich angesehenen und damit zumindest im Sinne des No-miracles-Arguments realistisch interpretierbaren Theorien einen Problemhorizont zu eröffnen, der gemeinhin als "pessimistische Metainduktion" bezeichnet wird. Diese pessimistische Metainduktion, eine Übertragung der kritischen Erfahrungen aus der Wissenschaftsgeschichte auf die heutige Situation der Wissenschaft, besteht in der ernstzunehmenden Befürchtung, dass sich die heutige Situation hinsichtlich der Naturbeschreibung aufgrund neuer Entwicklungen innerhalb der Wissenschaft sehr schnell in der gleichen Weise wie frühere, zu ihrer Zeit gültige und für richtig angesehene Beschreibungsvarianten als Luftschloss herausstellen könnte. Hilary Putnam hat die pessimistische Metainduktion einmal als "Alptraum des Realisten" bezeichnet und folgendermassen charakterisiert:

"What if all the theoretical entities postulated by one generation (molecules, genes, etc. as well as electrons) invariably 'don't exist' from the standpoint of later science? [...] One reason this is a serious worry is that eventually the following metainduction becomes compelling: just as no term used in the science of more than 50 (or whatever) years ago referred, so will turn out that no term used now (except maybe observation-terms if there are such) refers." (Putnam (1984) 145)

Die pessimistische Metainduktion hat nicht zuletzt einem ganzen Spektrum anti-realistischer Positionen Vorschub geleistet. So machen etwa die instrumentalistischen Sichtweisen hinsichtlich unserer empirischen Wissenschaft - neben der schon von David Hume hervorgehobenen, grundsätzlichen induktiven Unterbestimmtheit empirisch-wissenschaftlicher Theorien 
- vor allem die Erfahrungen mit der Wissenschaftsgeschichte und die aus den stattgefundenen Wissenschaftsumbrüchen heraus motivierbare pessimistische Metainduktion für sich geltend. Spätestens unsere Erfahrung mit Wissenschaftsumbrüchen haben uns, dem Instrumentalismus zufolge, gelehrt, die von einer Theorie beschriebenen Szenarien nicht wortwörtlich zu nehmen und als deskriptive Widerspiegelung realer Gegebenheit zu werten. Die Erfahrungen mit der Wissenschaftsgeschichte sind für den Instrumentalisten auch gar nicht erstaunlich, da unsere empirisch-wissenschaftlichen Theorien auf der Grundlage der immer nur finiten, unvollständigen empirischen Daten, über die wir zu einem bestimmten Zeitpunkt verfügen, grundsätzlich induktiv unterbestimmt sind. Es können immer wieder neue empirische Daten hinzukommen, die uns gänzlich andere Theorien nahelegen. Dem Instrumentalismus zufolge sind unsere wissenschaftlichen Theorien nichts mehr als Instrumente zur ökonomischen Zusammenfassung der beobachtbaren Phänomenologie, die, wenn sie ausreichend effektiv sind, uns vielleicht sogar streckenweise in die Lage versetzen, Vorhersagen $\mathrm{zu}$ treffen, die sich schliesslich empirisch bestätigen lassen. Die von einer Theorie postulierten Entitäten sind nur als Teil einer instrumentellen Struktur zu sehen, deren Bezug ausschliesslich durch die zugängliche Phänomenologie definiert ist. Es gibt nach Auffassung des Instrumentalismus keinen Grund davon auszugehen, dass diese Entitäten tatsächlich Gegenstücke in der Realität aufweisen. Insofern machen empirisch-wissenschaftliche Theorien keine wahren Aussagen über die von ihnen postulierten Entitäten.

Diese Auffassung deckt jedoch das Spektrum der anti-realistischen Reaktionen auf die pessimistische Metainduktion keineswegs ab. Bas Van Fraassen etwa kommt in seinem "Konstruktiven Empirismus" ${ }^{384} \mathrm{zu}$ einem etwas anderen Bild hinsichtlich unserer erfolgreichen empirisch-wissenschaftlichen Theorien als der Instrumentalismus, obwohl er in gleicher Weise die pessimistische Metainduktion und die induktive Unterbestimmtheit unserer empirisch-wissenschaftlichen Theorien als argumentative Ausgangsbasis wählt. Nach Van Fraassens Auffassung haben theoretische Entitäten unter Umständen vielleicht sogar eine Referenz in der Welt und Aussagen über theoretische Entitäten kommt vielleicht sogar ein Wahrheitswert zu. Aber dieser Wahrheitswert ist, ebenso wie die Referenz der theoretischen Terme, grundsätzlich nicht epistemisch zugänglich. Er ist auf der Grundlage empirischer Daten nicht erschliessbar. Van Fraassen zufolge

$384 \quad$ Siehe Van Fraassen (1980). 
sind unsere besten Theorien folglich nicht notwendigerweise wahr. Unsere besten Theorien können bestenfalls empirisch adäquat sein.

Wollte man nun annehmen, der Stringansatz sei, wenn er sich erst einmal durch unabhängige empirische Bestätigungen als erfolgreich erwiesen haben sollte, aufgrund der sich jetzt schon mit ihm abzeichnenden Auflösung jeglicher objekthafter Ontologie ${ }^{385}$ vielleicht ohne weiteres mit anti-realistischen Positionen, wie dem Instrumentalismus oder dem Konstruktiven Empirismus, verträglich, so stehen dem gewichtige Einwände entgegen:

Der Stringansatz bemüht sich, im Gegensatz zum Standardmodell, mit dem nur eine konzeptionelle, nicht aber eine nomologische Vereinheitlichung hinsichtlich der elektroschwachen und der starken Wechselwirkung erreicht wird, um eine nomologische Vereinigung aller Wechselwirkungen einschliesslich der Gravitation. Dass er diese nomologische Vereinigung von seiner formalen Konzeption her grundsätzlich leistet, ist, wie schon wiederholt betont, nicht etwa eine von vielen Eigenschaften, die der Stringansatz für sich geltend machen könnte, sondern es ist die zentrale Tatsache, die ihn überhaupt als mathematisches Konstrukt mit physikalischen Ambitionen ins Spiel gebracht hat und dort hält.

Der Stringansatz weist somit als seine zentrale und wesentlichste Motivation die Einbettung in das Programm einer nomologischen Vereinigung auf. Die Realisierung des nomologischen Vereinigungsprogramms ist aber letztlich nur vor dem Hintergrund einer realistischen Sichtweise ein erstrebenswertes Ziel. Der Instrumentalismus kommt sehr gut ohne nomologische Vereinigung aus und bietet für eine solche bestenfalls die unzureichende Motivation einer Ökonomisierung theoretischer Instrumentarien, die genauso gut im Kontext weniger ambitionierter und weniger metaphysisch beladener Zielsetzungen angestrebt werden kann. Wenn sich eine nomologische Vereinigung schliesslich erreichen lassen sollte, wäre sie dem Instrumentalismus vermutlich höchst suspekt. Das Programm der nomologischen Vereinheitlichung wird - ebenso wie die vor seinem Hintergrund erzielten Resultate in der physikalischen Theorienbildung - nie über seine metaphysischen Konnotationen und seine Verankerung im Realismus hinwegtäuschen können. 
Auch eine unabhängige empirische Bestätigung würde nichts daran ändern, dass der Stringansatz seine zentralen und massivsten Motivationen weiterhin aus diesem Programm schöpft. Hier ist es gerade die gerne vom Instrumentalismus ins Feld geführte, grundsätzliche empirische Unterbestimmtheit physikalischer Theorien, die nicht zuletzt zur Folge hat, dass eine empirische Bestätigung des Stringansatzes nicht etwa zu einer völligen Umkehrung dieses Motivationsverhältnisses führen kann. Das Programm einer nomologischen Vereinigung würde sich mit dem Erfolg des Stringansatz, vor allem mit seiner empirischen Bestätigung, nicht etwa definitiv als für die Naturbeschreibung angemessen beweisen lassen; es wäre vielmehr weiterhin ein von empirischen Belangen wenig tangiertes, metaphysisches Programm, das für den Stringansatz die entscheidende Motivation liefert und diesen aus einem Spektrum von möglichen theoretischen Szenarien auswählt und auszeichnet.

$\mathrm{Zu}$ den grundsätzlichen Alternativen innerhalb dieses Spektrums zählen etwa auch stückweise anwendbare, empirisch adäquate, effektive Theorien, die für sich nicht den nomologischen Vereinheitlichungsgedanken geltend machen. Irgendein Flickenteppich effektiver Theorien wäre als Alternative zur umfassenden nomologischen Vereinigung sicherlich immer erreichbar. Für den Instrumentalismus und den konstruktiven Empirismus wäre ein solcher Flickenteppich effektiver Theorien ohne weiteres akzeptabel, solange er empirisch adäquat ist. Vielleicht würden Instrumentalisten und konstruktive Empiristen zwecks Ökonomisierung ihrer theoretischen Instrumentarien nach konzeptionell einheitlicheren Theorien suchen; aber erst der Realismus bietet eine ausreichende Motivation, eine umfassende, nomologisch einheitliche Alternative für erstrebenswert zu erachten. Der nomologische Vereinheitlichungsgedanke als metaphysisches Konzept, welches den Stringansatz erst als physikalisch interessantes Konstrukt ausweist, ist letztlich nur vor dem Hintergrund einer realistischen Sichtweise hinsichtlich des Verhältnisses von Natur und Naturbeschreibung ausreichend motiviert.

Aber auch unabhängig davon dürfte es ohnehin ziemlich schwierig sein, den Stringansatz instrumentalistisch zu deuten. Er könnte nur in einem hochtheoretischen Sinne als Instrument zur Ökonomisierung der Phänomenologie gesehen werden. Eine solche theoretische Hochrüstung ist im Rahmen des Instrumentalismus kaum motivierbar. Ein einfacher handhabbares Instrumentarium aus effektiven Theorien mit einer grösseren Nähe 
zur Empirie ist für den Instrumentalismus nicht nur völlig ausreichend, sondern naheliegender. Eine aus dem Programm einer nomologischen Vereinheitlichung heraus motivierte theoretische Struktur mit maximaler Entfernung von der Phänomenologie, die hier gerade effektiv zusammengefasst werden soll, erscheint vor dem Hintergrund des Instrumentalismus kaum plausibel. Und solange der Stringansatz noch über keinen von seinen Vorgängertheorien unabhängigen empirischen Gehalt verfügt, ist er für den Instrumentalismus ohnehin völlig uninteressant.

Wenn also die angezielte nomologische Vereinigung die zentrale, wenn nicht gar die einzige Motivation dafür ist, den Stringansatz als physikalisches Programm überhaupt ernst zu nehmen, und wenn das Konzept der nomologischen Vereinigung wiederum nur im Rahmen einer realistischen Konzeption hinreichend motivierbar ist und den Realismus letztlich schon voraussetzt, so setzt auch der Stringansatz implizit schon den Realismus voraus, um überhaupt seinen Anspruch, ein physikalisch relevantes Theorienkonstrukt zu sein, geltend machen zu können. Offensichtlich gibt es also keinen Ausweg: Der Stringansatz kann aufgrund seiner Motivation durch den nomologischen Vereinheitlichungsgedanken, wenn überhaupt, nur vor dem Hintergrund eines realistischen Verständnisses des Verhältnisses von Natur und Naturbeschreibung gesehen werden.

Wenn der Stringansatz den Realismus voraussetzt, heisst dies aber noch nicht notwendigerweise, dass er problemlos mit dem Realismus auskommt. Dies macht spätestens die sich mit ihm abzeichnende Auflösung einer objekthaften Ontologie deutlich.

Schon die Vorläufertheorien des Stringansatzes haben ein zum Teil ambivalentes Verhältnis zur Realismusfrage. Insbesondere die Quantenmechanik lässt im Spektrum der epistemischen und nicht-epistemischen Interpretationen ihres Formalismus gleichermassen Anklänge an den Realismus wie den Instrumentalismus aufscheinen. ${ }^{386}$ Für die Allgemeine Relativitätstheorie ist die Frage nach der realistischen Interpretationsmöglichkeit von Raumzeitpunkten angesichts der Translationsinvarianz der Minkowski-Raumzeit und des Loch-Arguments immer noch ungeklärt. ${ }^{387}$

\footnotetext{
386 Die Literatur zu dieser Problematik ist inzwischen unüberschaubar.

387 Siehe etwa Norton (1988), (2004), Earman / Norton (1987), Earman (1989), Butterfield (1989) sowie Rynasiewicz (1994).
} 
Der Stringansatz trägt nun einerseits diese Ambivalenz in Bezug auf die Realismusfrage, ursächlich insbesondere aus der Quantenmechanik transferiert, in sich weiter, andererseits verliert er vor dem Hintergrund nicht-realistischer Sichtweisen sehr schnell seine Existenzberechtigung, die im wesentlichen einzig und allein auf dem nomologischen Vereinheitlichungsgedanken beruht, der den Realismus schon als Motivationskontext voraussetzt. Hierin unterscheidet sich der Stringansatz von seinen Vorläufertheorien; letztere können es sich leisten, eine wesentlich ambivalentere Position zum Realismus einzunehmen, da sie über unabhängige Motivationen verfügen, die zu einem erheblichen Grade in empirischen Bestätigungsinstanzen fussen und nicht etwa ausschliesslich in metaphysischen Programmen, die nur vor dem Hintergrund des Realismus sinnvoll erscheinen.

Wenn aber der Stringansatz eine grundsätzlich realistische Auffassung aufgrund seiner Motivation durch den nomologischen Vereinheitlichungsgedanken voraussetzt, andererseits jedoch die Ambivalenz der Quantenmechanik zum Realismusproblem geerbt hat, mit welcher Form von Realismus ist der Stringansatz dann kompatibel? Und liefert diese auch schon eine ausreichende Basis für den nomologischen Vereinheitlichungsgedanken?

Ein Realismus hinsichtlich der vom Stringansatz postulierten Entitäten kommt aufgrund der mit ihm einhergehenden Auflösung jeglicher objekthafter Ontologie wohl kaum in Frage. Das muss aber nicht unbedingt ein Problem sein. Denn schon die pessimistische Metainduktion, die sich aus der Tatsache heraus motivieren lässt, dass Wissenschaftsumbrüche oft gerade mit Umbrüchen hinsichtlich der von den betroffenen Theorien postulierten Entitäten einhergehen, lässt gerade einen Realismus hinsichtlich dieser Entitäten ohnehin problematisch erscheinen. Aber welchen Elementen innerhalb eines theoretischen Entwurfs lässt sich ontischer Status zusprechen - zumindest unter der Voraussetzung, dass dieser Entwurf sich in unabhängiger Weise empirisch bestätigen lässt? Welche theoretischen Elemente lassen sich dann als solche mit einem direkten Gegenstück in der Realität auffassen? 
John Worrall hat mit dem "Strukturenrealismus" eine Sichtweise entwickelt, die vielleicht gerade eine Antwort auf diese Frage liefert. ${ }^{388}$

"According to Worrall, we should not accept full-blown scientific realism, which asserts that the nature of things is correctly described by the metaphysical and physical content of our best theories. Rather we should adopt the structural realist emphasis on the mathematical or structural content of our theories. [...] structural realism both (a) avoids the force of the pessimistic meta-induction (by not committing us to belief in the theory's description of the furniture or the world), and (b) does not make the success of science (especially the novel predictions of mature physical theories) seem miraculous (by committing us to the claim that the theory's structure, over and above its empirical content, describes the world)." (Ladyman (1998) 409f)

Der Strukturenrealismus ist ein Realismus hinsichtlich der von empirisch erfolgreichen Theorien postulierten Strukturen, ohne eine realistische Sichtweise hinsichtlich der theoretisch postulierten Entitäten zu implizieren. Er ist also ein Realismus ohne Festlegung auf eine objekthafte Ontologie.

"This would seem to entail a corresponding shift from a metaphysics of objects, properties, and relations, to one that takes structure as primitive." (Ladyman (1998) 418)

Der Strukturenrealismus lässt sich somit als Reaktion auf die Gefährdung des Realismus durch die pessimistische Metainduktion verstehen. Er besteht gerade im Versuch der Vereinbarung des für den Realismus sprechenden No-miracles-Arguments mit unserer Erfahrung aus der Wissenschaftsgeschichte, dass Wissenschaftsumbrüche oft mit Umbrüchen hinsichtlich der objekthaften Ontologie einhergehen - der Erfahrung, die gerade die pessimistische Metainduktion evoziert und einen Entitätenrealismus fragwürdig werden lässt.

"In the debate about scientific realism, arguably the two most compelling arguments around are the 'no miracles' argument, and the

388 Siehe Worrall (1989) und Ladyman (1998). Eine Kritik findet sich in Psillos (1995), (1996) und (1999). Worrall selbst führt die Grundidee seines "Strukturenrealismus" auf Henri Poincaré zurück. 
'pessimistic meta-induction'. Unfortunately these pull in opposite directions (towards realism and antirealism respectively). In an attempt to break this impasse, and have 'the best of both worlds', John Worrall introduced structural realism (although he attributes its original formulation to Poincaré." (Ladyman (1998) 409)

Als Argument für seinen Strukturenrealismus führt Worrall die These ins Feld, dass die wesentlichsten mathematischen Strukturen, die von empirisch-wissenschaftlichen Ansätzen zur Beschreibung der Wirklichkeit zur Anwendung gebracht werden, sich über Wissenschaftsumbrüche hinweg wesentlich stabiler verhalten als die von den jeweilig betroffenen Theorien postulierten Entitäten. Die Strukturen verändern sich nach Worralls Auffassung über Wissenschaftsumbrüche hinweg eher kumulativ im Sinne einer struktureller Erweiterung anstatt einer strukturellen Ablösung.

"[...] the continuity in science is in the intension not the extension of its concepts." (Ladyman (1998) 418)

Diese Aufrechterhaltung und Erweiterung der Strukturen, an der die wissenschaftshistorische Kontinuität der Naturbeschreibung festgemacht wird, wird nicht zuletzt als Zeichen für die Konvergenz der physikalischen Theorienbildung hinsichtlich dieser Strukturen gewertet. Demzufolge kommt diesen Strukturen eine zumindest approximative Wahrheit zu.

\begin{abstract}
"[...] from the perspective of the semantic approach we do not expect the high-theoretical structure to be a law-like description of the world, rather we envisage a complex hierarchy of models or structures employed by the theory. [...] abstract theoretical structure plays an important role in the development of low-level models of the phenomena, and some of the most profound novel predictions of science are directly obtained from highly theoretical models. [...] Hence, the most theoretical parts of a theory, the abstract mathematical structures it employs at the greatest level of generality, must have some grip on reality. It is clear that the 'grip on reality' in question must go beyond a correct description of the actual phenomena to the representation of modal relations between them." (Ladyman (1998) 418)
\end{abstract}

Das No-miracles-Argument als entscheidende Komponente des Strukturenrealismus wird aber per se erst wirksam, wenn eine ausreichende empiri- 
sche Bestätigung für die entsprechende Theorie vorliegt. Und diese empirische Bestätigung muss gegenüber der in die Theorienbildung eingeflossenen Empirie, insbesondere dem schon für die Vorläufertheorien feststellbaren empirischen Gehalt, autonom sein.

"Notice, by the way, that the argument requires the empirical success of a theory to be understood in a particular way. Not every empirical consequence that a theory has and which happens to be correct will give intuitive support for the idea that the theory must somehow or other have latched onto the 'universal blueprint'. Specifically, any empirical consequence which was written into the theory post hoc must be excluded. Clearly it is no miracle if a theory gets right a fact which has already known to hold and which the theory had been engineered to yield." (Worrall (1989) 101)

Es reicht also nicht, wenn eine neue Theorie für sich einen empirischen Gehalt geltend machen kann, der schon mit ihren Vorläufertheorien gegeben war. Es reicht also nicht, wenn der Stringansatz glaubwürdig machen kann, dass er die Allgemeine Relativitätstheorie und die von den Quantenfeldtheorien beschriebene Dynamik - und mithin die in diese schon eingeflossene Empirie - reproduzieren kann. Erst eine davon unabhängige empirische Bestätigung stellt eine Verbindung zwischen der neuen Theorie und der Wirklichkeit, die zu beschreiben sie vorgibt, her.

"Sal - I am reading a book, written long ago, where I just found this phrase: '... perchè i nostri discorsi hanno essere sopra un mondo sensibile, e non sopra un mondi di carta.' Roughly: '... our arguments have to be about the world we experience, not about a world made of paper'. None of those theories is connected to the world, as far as we know." (Rovelli (2003) 5)

Erst, wenn eine unabhängige empirische Bestätigung vorliegt, kann das No-miracles-Argument im Sinne des Strukturenrealismus wirksam werden. Und erst dann lässt sich die fundamentale Frage des Strukturenrealismus stellen, welche Strukturen ernst zu nehmen sind. Eine Antwort auf diese Frage wird dann eine genaue Analyse der entsprechenden, nun in unabhängiger Weise empirisch bestätigten Theorie und ihrer Form der Ankopplung an die Empirie erfordern. 
Die Frage nach den diesbezüglich relevanten Strukturen ist also für den Stringansatz zum heutigen Stand sicherlich nicht adäquat beantwortbar. Nach dem, was im vorausgehenden Kapitel hinsichtlich der Uneindeutigkeit der von den diversen Theorieszenarien entworfenen strukturellen Gegebenheiten zu konstatieren war, bleibt es erst einmal völlig unklar, welche Strukturelemente ontisch gedeutet werden können und welche als formale, mathematische Überschussstruktur zu sehen sind. Die Frage der ontischen Deutbarkeit theoretischer Strukturen kann sich im Falle des Stringansatzes nicht nur in keiner Weise auf irgendeine empirische Instanz der Überprüfung einer spezifischen Theorie stützen; vielmehr gibt es letztlich noch nicht einmal eine solche ausgereifte, spezifische Theorie.

Und es ist immerhin fraglich, ob der Strukturenrealismus mit multiplen (alternativen) Strukturen zurechtkommt, wie sie augenblicklich für den Stringansatz vorliegen. Es müsste sich auf einer vielleicht noch auszumachenden Darstellungsebene schon die durchgängige (und nicht nur parameterbereichsspezifische) Äquivalenz der entsprechenden theoretischen Szenarien hinsichtlich ihrer strukturellen Implikationen nachweisen lassen, um diese Schwierigkeit zu beheben. Aber im Stringansatz sieht es bisher eher danach aus, als ob sich im Rahmen der Dualitätsbeziehungen gerade phänomenologische Äquivalenzen zwischen Szenarien aufzeigen lässt, die sich in Hinsicht auf ihre struktureller Festlegungen und Gegebenheiten völlig unterschiedlich gestalten. ${ }^{389}$ Die phänomenologische Äquivalenz

389 Richard Dawid hat hinsichtlich des Stringansatzes und der Entwicklungen innerhalb der modernen Physik, die zu ihm hingeführt haben, einen (vor diesem Hintergrund multipler Strukturen sehr problematischen) "Consistent Structure Realism" geltend gemacht. Siehe Dawid (2003) und (2004). Dawid zufolge zeichnet die Einzigartigkeit einer durch Konsistenz erzwungenen theoretischen Struktur diese Struktur als einzig mögliche Struktur der Welt aus.

"In a conventional theoretical setting hypothetical theoretical models must be distinguished from the one theoretical model that is actually realised in our world. Real existence thus represents a quality that has to be attributed to a certain scenario based on observation. Once the physical description has become theoretically unique however, consistence becomes synonymous with real existence. No additional quality has to be awarded to the consistent scheme in order to show its distinction." (Dawid (2003) 30)

Die empirische Bestätigung eines sich durch Konsistenz auszeichnenden theoretischen Konstrukts wird nach Dawids Einschätzung irrelevant, sobald sich zeigen lässt, dass dieses Konstrukt das Möglichkeitsspektrum vollständig abdeckt. Dies sieht Dawid für den Stringansatz als vollständiger Erfassung aller strukturellen Möglichkeiten auf der Ebene einer fundamentalen Weltbeschreibung gegeben. 
zwischen strukturell verschiedenen Szenarien widerspricht letztlich dem Grundgedanken des Strukturenrealismus. Sie könnte sich jedoch schliesslich als ein Artefakt der zur Zeit verwendeten perturbativen Prozeduren herausstellen, das sich vielleicht im Rahmen weiterer Metamorphosen des Stringansatzes überwinden lässt. Solange keine Verbindung des Stringansatzes mit der Empirie erreicht wird, ist die Problematik der konkreten Vereinbarkeit des Stringansatzes mit dem Strukturenrealismus aber ohnehin noch nicht akut.

Was sich aber jetzt schon für den Fall abzeichnet, dass der Stringansatz irgendwann einmal über unabhängige empirische Bestätigungen verfügen sollte, ist folgendes: Da der Stringansatz aufgrund seiner nomologischen Vereinheitlichungsabsicht nur sinnvoll im Rahmen einer realistischen Konzeption gesehen werden kann, der Entitätenrealismus aber infolge der mit der Entwicklung der Stringtheorien einhergehenden Auflösung jeglicher objekthafter Ontologie kaum in Frage kommt, kann es, wenn überhaupt, nur irgendeine Form von Strukturenrealismus sein, vor dessen Hintergrund der Stringansatz hinsichtlich der Deutung seiner Aussagen gesehen werden muss.

"If you knock at all doors you can think of and precisely one of them opens, chances are good that you are on the right track if you enter. Scientists working on quantum gravity have thought about all currently conceivable options, including those which drop fundamental physical principles." (Dawid (2004) 6)

Die für eine solche Beschreibungsebene relevanten empirischen Komponenten haben sich nach Dawids Einschätzung schon in früheren Entwicklungsschritten der Theorienbildung in Form von physikalischen Prinzipien in die Theorienkonstrukte eingeschrieben. Über diese Prinzipien lässt sich nun, Dawid zufolge, auf der einmal erreichten fundamentalsten Beschreibungsebene die vollständige Abdeckung des Möglichkeitsspektrums begründen.

"Theoretical physics might be seen as the science that finds out which structural features are impossible. The impossibility of an ever increasing number of simple structural solutions leads to more elaborate solutions, enforces the modification of foundational postulates and thereby reveals the complexity of reality." (Dawid (2003) 31)

$\mathrm{Ob}$ jedoch der sich in die Theorienbildung über die jeweiligen Vorläufer einschreibende empirische Gehalt zu einer ausreichenden und eindeutigen Fixierung relevanter Prinzipien führt, ist sehr fraglich. Ob der Stringansatz das konsequente und einzig mögliche Ergebnis eines solchen Prozesses ist, ist noch fraglicher. Das in Kapitel 5. erörterte Kontingenzproblem lässt die Idee einer unmittelbaren Kopplung von Konsistenz, Einzigartigkeit und empirischer Adäquatheit als höchst zweifelhaft erscheinen. 
Das hierbei grundlegendste (und von den zur Zeit für den Stringansatz vorliegenden multiplen Strukturen im wesentlichen unabhängige) Argument für die Unvereinbarkeit mit einem Entitätenrealismus ist in der Tatsache zu sehen, dass sich, der Konzeption des Stringansatzes als intendierter fundamentalster physikalischer Theorie zufolge, alles, was sich auf der von ihm als basal ausgezeichneten dynamischen Ebene abspielt, nicht mehr realistisch im Sinne eines raumzeitlichen Agierens und Interagierens von objekthaften Entitäten interpretieren lässt. Strings können keinesfalls als räumlich strukturierte und räumlich ausgedehnte Entitäten verstanden werden. Sie stellen, wenn sie tatsächlich die basalen Einheiten sein sollten, nur eine mathematische Metapher für ein Etwas dar, das auf der Grundlage seiner Freiheitsgrade und mittels seiner Wirkungen auf spezifische Art und Weise dynamisch in Erscheinung treten kann.

Wenn die fundamentalste Ebene des Stringansatzes mit der Planck-Ebene zusammenfällt, so sprechen auch die zuvor ${ }^{390}$ schon diskutierten Argumente für eine fundamentale Diskretheit der Raumzeit und der physikalischen Strukturen auf der Planck-Ebene (Entropie Schwarzer Löcher, holographisches Prinzip) gerade für diese Tatsache. Es kann für diesen Bereich keinen Objektbegriff mit raumzeitlichen Konnotationen mehr geben. Es ist bestenfalls an eine mathematische Struktur zu denken, auf deren Grundlage sich erst Raumzeit und Objekthaftigkeit konstituieren.

Diese Argumente sprächen für den Fall, dass der Stringansatz sich in weiteren Metamorphosen tatsächlich einmal auf eine eindeutige Struktur festlegen lassen sollte und es gelingen sollte, aus dieser Struktur unabhängige, empirisch relevante Vorhersagen abzuleiten und diese empirisch zu bestätigen, gleichzeitig für eine ontische und gegen eine rein epistemische Deutung des Strukturenrealismus.

Im Sinne einer solchen ontischen Deutung des Strukturenrealismus sind es die Strukturen selbst (und nicht etwa irgendwelche Entitäten, die vielleicht unerschliessbar hinter den empirisch-wissenschaftlich erschliessbaren Strukturen stehen), die letztendlich die Realität ausmachen. Dies würde mit Sicht auf den Stringansatz bedeuten, dass die zunehmende Auflösung einer entitätenorientierten, objekthaften Ontologie im Rahmen der Entwicklung der modernen Physik, die im Stringansatz vielleicht zu ihrem Abschluss kommt, einer Annäherung unserer physikalischen Naturbeschreibung an

$390 \quad$ Siehe Kap. 6. 
die tatsächlichen Gegebenheiten einer Realität entspricht, die sich in letzter Instanz durch Strukturen und nicht durch Entitäten auszeichnet.

Im Rahmen einer nur epistemischen Deutung des Strukturenrealismus wären die Strukturen unserer fundamentalsten, empirisch bestätigten Theorien das, was uns von der Natur im Rahmen unserer epistemischen Bemühungen (augenblicklich oder grundsätzlich) zugänglich ist. Ob es irgendwelche fundamentalen Entitäten hinter diesen Strukturen gibt, ist im Sinne der epistemischen Variante des Strukturenrealismus nicht entscheidbar, da uns zur Beantwortung dieser Frage die epistemischen Erschliessungsmöglichkeiten fehlen. Im Sinne einer solchen epistemischen Variante des Strukturenrealismus könnte die zunehmende Auflösung einer entitätenorientierten, objekthaften Ontologie im Rahmen der Entwicklung der modernen Physik ohne weiteres ein Artefakt sein, das aus einer auf bestimmte konzeptionelle Modalitäten oder auf eine spezifische modelltheoretische Grundlage festgelegten Theorienbildung resultiert. Die Auflösung der objekthaften Ontologie müsste aber noch nicht notwendigerweise als Konsequenz einer Annäherung unserer physikalischen Naturbeschreibung an die tatsächlichen Gegebenheiten der Realität gesehen werden.

Alle diese Überlegungen besitzen jedoch angesichts der heutigen Theoriensituation im Bereich der Quantengravitation bestenfalls Vorläufigkeitsstatus. Eine abschliessende Entscheidung hinsichtlich der Stellung des Stringansatzes zum Realismus ist zur Zeit noch in keiner Weise möglich. 
